\title{
Exposure to styrene and chronic health effects: mortality and incidence of solid cancers in the Danish reinforced plastics industry
}

\author{
Henrik A Kolstad, Knud Juel, Jørn Olsen, Elsebeth Lynge
}

\begin{abstract}
Objectives-To study the occurrence of non-malignant diseases and solid cancers in workers exposed to styrene in the Danish reinforced plastics industry.

Methods-A11 36610 workers of 386 reinforced plastics companies and 14293 workers not exposed to styrene from similar industries were followed up from 1970 to 1990 . This industry is characterised by simple exposure conditions, exposure to high concentrations of styrene, and a high proportion of small companies, and the exposure assessment was based on experts' classification on a company level. The mortality from nonmalignant causes and the incidence of solid cancers were compared with the national rates. Poisson models were used for internal comparisons.
\end{abstract}

Results-A total of 3031 deaths and 1134 newly diagnosed cases of solid cancer were reported in the workers in the reinforced plastics industry. In companies where $50 \%$ or more of the workers produced reinforced plastics an increased mortality rate ratio (MRR) for degenerative disorders of the nervous system (multiple sclerosis, parkinsonism, and motor neurone disease; MRR 1.8, 16 cases, 95\% confidence interval (95\% CI) $0.9-3 \cdot 8)$ and an increased incidence rate ratio (IRR) for pancreatic cancer (IRR $2 \cdot 2,17$ cases, 95\% CI 1·1-4.5) was found. For both disease categories increased occurrence was also found among long term workers, workers of the 1960 s (the period with the highest exposure to styrene), and workers with a latent period of more than 10 years after the start of employment. No other non-malignant diseases or solid cancers showed these patterns.

Conclusion-The findings have to be interpreted with caution, due to the company based exposure assessment, but the possible association between exposures in the reinforced plastics industry, mainly styrene, and degenerative disorders of the nervous system and pancreatic cancer, deserves attention.

(Occup Environ Med 1995;52:320-327)

Correspondence to: Dr Henrik A Kolstad, Institute of Epidemiology and Social Medicine, University of Aarhus, Building 2C, Nørrebrogade 44, DK-8000 Århus C.

Accepted 30 January 1995
Keywords: pancreatic neoplasms; degenerative nervous diseases; styrene; cohort study
Styrene is an organic solvent and it is one of the most important composites of plastics and synthetic rubber. In 1992 about 14 million tonnes of styrene were used globally. ${ }^{1}$ Styrene is found at low concentrations in the ambient air and in tobacco smoke. ${ }^{2}$ Much higher exposure occurs in industrial plants that produce styrene monomer, styrene-butadiene plastics, or polystyrene. ${ }^{1}$ The highest exposures have been found in the reinforced plastics industry in which styrene is the predominant exposure but acetone is also often used. ${ }^{3}$ Both are organic solvents and the industry offers an important setting for the study of health effects of organic solvents, mainly styrene.

Acute neurotoxic effects of styrene have been found as symptoms of the central nervous system; such as electroencephalographic abnormalities, delayed psychomotor reaction, and impairment of colour vision. ${ }^{4-7}$ Whether styrene can cause chronic effects on the central nervous system is a matter of debate.$^{8}$ It has, however, been proposed that long lasting exposure to organic solvents in high doses may cause multiple sclerosis, ${ }^{9}$ parkinsonism, ${ }^{10}$ motor neurone disease, ${ }^{11}$ and neuropsychiatric disability. ${ }^{12}$ Cases of congestive cardiomyopathy in glue sniffers ${ }^{13}$ have been associated with high exposure to organic solvents. Occupational exposure to hydrocarbons has been linked with chronic pancreatitis ${ }^{14}$ and with chronic glomerulonephritis in several case-control studies, ${ }^{15}{ }^{16}$ but not in all. ${ }^{17}$

Cytogenetic effects have been found in workers exposed to high concentrations of styrene ${ }^{18}$ as have moderately increased occurrences of lymphomas and leukaemia. ${ }^{19-21}$ Not all the studies have found these effects. ${ }^{22}$ No consistently increased mortality from solid cancers in workers exposed to styrene has been found in previous studies. Styrene is classified by the International Agency for Research on Cancer (IARC) as a possible human carcinogen. ${ }^{23}$

The aim of our study was to report the results of health surveillance of a large group of workers exposed to styrene on the mortality of non-malignant diseases and the incidence of solid cancers. The occurrence of lymphohaematopoietic malignancies in this population has been reported previously. ${ }^{2021}$

Materials and methods

Five hundred and fifty two companies that have produced reinforced plastics in Denmark since the early 1960 s were identified. Most of these were boat yards or manufacturers of 
Table 1 Non-malignant causes of death that may be associated with exposure to organic solvents: data from a cohort of men exposed to styrene in the Danish reinforced plastics industry 1970 to 1990

\begin{tabular}{|c|c|c|c|c|}
\hline \multirow[b]{3}{*}{ Cause of death (ICD-8 code) } & \multicolumn{4}{|l|}{ Number of cases } \\
\hline & \multirow{2}{*}{$\begin{array}{l}\text { By underlying } \\
\text { cause of deat } h^{\star}\end{array}$} & \multicolumn{3}{|c|}{ By underlying and contributory cause of death $\dagger$} \\
\hline & & Exposed $\ddagger$ & Unexposed $\ddagger$ & Total \\
\hline \multicolumn{5}{|c|}{ Degenerative disorders of the nervous system: } \\
\hline Multiple sclerosis (340) & 4 & 6 & 1 & 7 \\
\hline Parkinsonism (342) & 6 & 18 & 6 & 24 \\
\hline Other diseases of brain (347) & 10 & 17 & 7 & 24 \\
\hline Motor neurone disease (348) & 4 & 4 & & \\
\hline \multirow{2}{*}{\multicolumn{5}{|c|}{ Degenerative disorders of the myocardium: }} \\
\hline & & & & \\
\hline Myocarditis (422) & 3 & 2 & 1 & 3 \\
\hline Cardiomyopathy (425) & 10 & 10 & 3 & 13 \\
\hline Other myocardial insufficiency (428) & 6 & 9 & 3 & 12 \\
\hline Total & 19 & 21 & 7 & 28 \\
\hline Pancreatitis (577) & 11 & 32 & 5 & 37 \\
\hline Glomerulonephritis (580-584) & 6 & 8 & 3 & 11 \\
\hline
\end{tabular}

*Cases included in external analysis; †cases included in internal analysis; łexposed = employed in company producing reinforced plastics; unexposed $=$ employed in company with no exposure to styrene.

containers produced by hand lamination. The 53847 male workers ever employed between 1964 and 1988 and alive on 1 January 1970 , were found by linkage of unique company and personal identification numbers in a national pension fund. Two dealers of plastic raw materials independently reviewed the production of all companies and classified them as (a) producing reinforced plastics with $50 \%$ or more of the workforce involved in the production (287 companies, 12862 employees) or (b) producing reinforced plastics with less than $50 \%$ of the work force involved in the production (99 companies, 23748 employees), or (c) not producing reinforced plastics (84 companies, 14293 employees). Eighty two companies unknown to the dealers (2944 employees) were excluded. The dealers' classification showed a high correlation with comparable measures reported by 368 employers who answered a postal questionnaire $(\kappa 0 \cdot 72)$. The analysis was based on the dealers' estimates as they were the most complete.

No data on individual exposure or job titles was available, but employees of companies with less than $50 \%$ of the workers involved with reinforced plastics were classified as of probable low exposure to styrene, whereas employees of companies with $50 \%$ or more of the workers involved with reinforced plastics were classified as of probable high exposure. Of all the reinforced plastics companies, $92 \%$ had an average work force of less than 50 employees corresponding to $44 \%$ of all employees of the reinforced plastics companies. In companies with a probable high exposure $77 \%$ of all workers were employed in companies with an average work force below 50 workers. Duration of employment was estimated from the payments recorded for each employee in the pension fund (for workers with reinforced plastics only employment in companies that produced reinforced plastics was counted). Details on the identification and classification of the study population have been published earlier. ${ }^{2124}$

The exposure concentrations of styrene in work room air of the Danish reinforced

Table 2 Non-malignant causes of death among men employed in the Danish reinforced plastics industry and similar industries 1970 to 1990 (expected numbers are based on national rates)

\begin{tabular}{|c|c|c|c|c|c|c|}
\hline \multirow[b]{2}{*}{ Cause of death (ICD-8 code) } & \multicolumn{3}{|c|}{ Reinforced plastics industry } & \multicolumn{3}{|c|}{ Industries with no exposure to styrene } \\
\hline & Obs & $\operatorname{Exp}$ & $\operatorname{SMR}(95 \% C I)$ & Obs & $\operatorname{Exp}$ & $S M R(95 \% C I)$ \\
\hline All deaths & 3031 & $2754 \cdot 3$ & $1 \cdot 10(1 \cdot 06-1 \cdot 14)$ & 1453 & $1241 \cdot 2$ & $1 \cdot 17(1 \cdot 11-1 \cdot 23)$ \\
\hline Infectious diseases $(000-136)$ & 15 & $19 \cdot 5$ & $0.77(0.43-1.27)$ & 7 & $8 \cdot 3$ & $0.84(0.34-1.74)$ \\
\hline Endocrine system (240-279) & 52 & $52 \cdot 5$ & $0.99(0.74-1.30)$ & 22 & $22 \cdot 3$ & $0.99(0.62-1.49)$ \\
\hline Haematological system $(280-289)$ & 3 & $3 \cdot 3$ & $0.91(0 \cdot 16-4 \cdot 82)$ & 2 & 1.5 & $1 \cdot 33(0 \cdot 16-4 \cdot 82)$ \\
\hline Mental disorders $(290-315)$ & 25 & $23 \cdot 3$ & $1.07(0.69-1.58)$ & 14 & $9 \cdot 6$ & $1.46(0.80-2 \cdot 45)$ \\
\hline Alcoholism (303) & 21 & $17 \cdot 6$ & $1.19(0.74-1.82)$ & 10 & $6 \cdot 8$ & $1.47(0.71-2 \cdot 70)$ \\
\hline Nervous system (320-389) & 29 & $34 \cdot 5$ & $0.84(0.56-1.21)$ & 11 & $14 \cdot 8$ & $0.74(0.37-1.33)$ \\
\hline $\begin{array}{l}\text { Degenerative disorders of the } \\
\text { nervous system }(340,342,347,348)\end{array}$ & & & & & & \\
\hline $\begin{array}{l}\text { nervous system }(340,342,347,348) \\
\text { Circulatory system }(390-458)\end{array}$ & 18 & $\begin{array}{r}19 \cdot 1 \\
1053 \cdot 8\end{array}$ & $\begin{array}{l}0.94(0.56-1.50) \\
1.03(0.97-1.09)\end{array}$ & 6 & $\begin{array}{r}7 \cdot 3 \\
496 \cdot 2\end{array}$ & $\begin{array}{l}0.82(0.30-1.79) \\
1.14(1.04-1.23)\end{array}$ \\
\hline $\begin{array}{l}\text { Circulatory system }(390-458) \\
\text { Ischaemic heart disease }(410-414)\end{array}$ & $\begin{array}{r}1085 \\
787\end{array}$ & $\begin{array}{r}1053 \cdot 8 \\
741 \cdot 5\end{array}$ & $\begin{array}{l}1.03(0.97-1.09) \\
1.06(0.99-1.13)\end{array}$ & $\begin{array}{l}564 \\
396\end{array}$ & $\begin{array}{l}496 \cdot 2 \\
346 \cdot 2\end{array}$ & $\begin{array}{l}1.44(1.04-1.23) \\
1.14(1.03-1.26)\end{array}$ \\
\hline $\begin{array}{l}\text { Degenerative disorders of the } \\
\text { myocardium }(422,425,428)\end{array}$ & & & & & & \\
\hline $\begin{array}{l}\text { myocardium }(422,425,428) \\
\text { Respiratory system }(460-519)\end{array}$ & 17 & $\begin{array}{r}9 \cdot 5 \\
150 \cdot 8\end{array}$ & $\begin{array}{l}1.79(1.04-2.87) \\
1.16(0.99-1.35)\end{array}$ & $\begin{array}{r}2 \\
78\end{array}$ & $\begin{array}{r}4 \cdot 1 \\
73 \cdot 0\end{array}$ & $\begin{array}{l}0.49(0.06-1.76) \\
1.07(0.84-1.33)\end{array}$ \\
\hline $\begin{array}{l}\text { Respiratory system (460-519) } \\
\text { Chronical respiratory diseases } \\
(490-493)\end{array}$ & 175 & $150 \cdot 8$ & $1.16(0.99-1.35)$ & & & \\
\hline $\begin{array}{l}\quad(490-493) \\
\text { Digestive system (520-577) }\end{array}$ & 114 & $105 \cdot 2$ & $1 \cdot 08(0.89-1 \cdot 30)$ & 54 & $49 \cdot 9$ & $1.08(0.81-1.41)$ \\
\hline $\begin{array}{l}\text { Digestive system (520-577) } \\
\text { Cirrhosis of the liver (571) }\end{array}$ & 118 & $123 \cdot 2$ & $0.96(0.79-1.15)$ & 60 & 53.9 & $1.11(0.85-1.43)$ \\
\hline $\begin{array}{l}\text { Cirrhosis of the liver (571) } \\
\text { Pancreatitis (577) }\end{array}$ & 70 & $69 \cdot 2$ & $1.01(0.79-1.28)$ & 40 & $27 \cdot 4$ & $1.46(1.04-1.99)$ \\
\hline $\begin{array}{l}\text { Pancreatitis (577) } \\
\text { Genitourinary system (580-629) }\end{array}$ & 10 & $7 \cdot 9$ & $1.27(0.61-2.33)$ & 1 & $3 \cdot 3$ & $0.30(0.01-1.69)$ \\
\hline Genitourinary system $(580-629)$ & 28 & $23 \cdot 4$ & $1 \cdot 20(0 \cdot 80-1 \cdot 73)$ & 7 & $11 \cdot 4$ & $0.61(0.25-1.27)$ \\
\hline $\begin{array}{l}\text { Glomerulonephritis (580-584) } \\
\text { Symptoms and ill defined conditions }\end{array}$ & 6 & $5 \cdot 5$ & $1.09(0.40-2.37)$ & 0 & $2 \cdot 3$ & $0.00(0.00-1.60)$ \\
\hline Symptoms and ill defined conditions & 128 & $101 \cdot 6$ & $1 \cdot 26(1 \cdot 05-1 \cdot 50)$ & 50 & $43 \cdot 7$ & $1 \cdot 14(0 \cdot 85-1 \cdot 51)$ \\
\hline $\begin{array}{l}\text { Àccidents, poisonings, and } \\
\text { violence (E800-E999) }\end{array}$ & & & & & & $1.49(1.30-1.67)$ \\
\hline $\begin{array}{l}\text { violence (E800-E999) } \\
\text { Suicide (E950-E959) }\end{array}$ & $\begin{array}{l}557 \\
238\end{array}$ & $\begin{array}{l}399 \cdot 5 \\
187 \cdot 1\end{array}$ & $\begin{array}{l}1.39(1.28-1.52) \\
1.27(1.12-1.44)\end{array}$ & $\begin{array}{r}241 \\
96\end{array}$ & $\begin{array}{r}102 \cdot 1 \\
74 \cdot 3\end{array}$ & $1.29(1.05-1 \cdot 58)$ \\
\hline
\end{tabular}


Table 3 Mortalities and mortality rate ratios (MRR) for selected non-malignant causes of death among men employed in the Danish reinforced plastics (RP) industry 1970 to 1990 (results of Poisson regression analysis with workers not exposed to styrene as controls)

\begin{tabular}{|c|c|c|c|c|c|c|c|c|c|c|c|c|}
\hline \multirow{3}{*}{$\begin{array}{l}\text { Cause of death and } \\
\text { employment } \\
\text { data }\end{array}$} & \multicolumn{12}{|c|}{ Exposure probability } \\
\hline & \multicolumn{3}{|c|}{ Unexposed controls } & \multicolumn{3}{|l|}{ Low } & \multicolumn{3}{|l|}{ High } & \multicolumn{3}{|c|}{ All RP workers } \\
\hline & Obs & Rate & $M R R(95 \% C I)$ & Obs & Rate & $M R R(95 \% C I)$ & Obs & Rate & $M R R(95 \% C I)$ & Obs & Rate & $M R R(95 \% C I)$ \\
\hline \multicolumn{13}{|c|}{ Degenerative disorders of the nervous system } \\
\hline $\begin{array}{l}\text { Year of first employment: } \\
>1970\end{array}$ & 3 & $2 \cdot 9$ & $1 \cdot 0$ & 6 & $9 \cdot 6$ & $1 \cdot 1(0 \cdot 3-4 \cdot 4)$ & 7 & $6 \cdot 2$ & $1 \cdot 8(0 \cdot 5-7 \cdot 1)$ & 13 & $4 \cdot 8$ & $1.4(0.4-4.9)$ \\
\hline$\leqslant 1970$ & 12 & $16 \cdot 5$ & $\begin{array}{l}1 \cdot 6(0 \cdot 4-6 \cdot 0) \\
1 \cdot 0\end{array}$ & 23 & $17 \cdot 6$ & $\begin{array}{l}2 \cdot 0(0 \cdot 6-7 \cdot 0) \\
1 \cdot 2(0 \cdot 6-2 \cdot 4)\end{array}$ & 9 & $23 \cdot 3$ & $\begin{array}{l}3 \cdot 1(0 \cdot 8-11 \cdot 8) \\
1 \cdot 8(0 \cdot 8-4 \cdot 3)\end{array}$ & 32 & 18.9 & $\begin{array}{l}2 \cdot 2(0 \cdot 7-7 \cdot 7) \\
1 \cdot 3(0 \cdot 7-2 \cdot 6)\end{array}$ \\
\hline $\begin{array}{l}\text { Duration of employment: } \\
<1 \mathrm{y}\end{array}$ & 6 & $5 \cdot 6$ & $1 \cdot 0$ & 8 & $4 \cdot 7$ & $0.9(0 \cdot 3-2 \cdot 7)$ & 8 & $7 \cdot 8$ & $1 \cdot 9(0 \cdot 6-5 \cdot 5)$ & 16 & $5 \cdot 8$ & $1 \cdot 3(0 \cdot 5-3 \cdot 2)$ \\
\hline$\geqslant 1 \mathrm{y}$ & 9 & $13 \cdot 0$ & $\begin{array}{l}1 \cdot 3(0 \cdot 4-3 \cdot 6) \\
1 \cdot 0\end{array}$ & 21 & $17 \cdot 4$ & $\begin{array}{l}1 \cdot 7(0 \cdot 7-4 \cdot 2) \\
1 \cdot 3(0 \cdot 6-2 \cdot 8)\end{array}$ & 8 & $16 \cdot 7$ & $\begin{array}{l}2.5(0.9-7 \cdot 4) \\
1.9(0.7-5 \cdot 0)\end{array}$ & 29 & $17 \cdot 2$ & $\begin{array}{l}1 \cdot 8(0 \cdot 7-4 \cdot 3) \\
1 \cdot 4(0 \cdot 7-3 \cdot 0)\end{array}$ \\
\hline $\begin{array}{l}\text { Time since first employment: } \\
<10 y\end{array}$ & 1 & 0.9 & $1 \cdot 0$ & 3 & $1 \cdot 8$ & $1.9(0.2-18 \cdot 2)$ & 3 & $3 \cdot 0$ & $3 \cdot 8(0 \cdot 4-36 \cdot 2)$ & 6 & $2 \cdot 2$ & $2.5(0.3-20.8)$ \\
\hline$\geqslant 10 \mathrm{y}$ & 14 & $20 \cdot 3$ & $\begin{array}{l}4 \cdot 6(0 \cdot 6-38 \cdot 4) \\
1 \cdot 0\end{array}$ & 26 & $20 \cdot 9$ & $\begin{array}{l}5 \cdot 3(0 \cdot 7-42 \cdot 7) \\
1 \cdot 1(0 \cdot 6-2 \cdot 1)\end{array}$ & 13 & $25 \cdot 5$ & $\begin{array}{l}8 \cdot 0(0 \cdot 9-66 \cdot 3) \\
1 \cdot 7(0 \cdot 8-3 \cdot 6)\end{array}$ & 39 & $22 \cdot 2$ & $\begin{array}{l}6 \cdot 4(0 \cdot 8-51 \cdot 2) \\
1 \cdot 2(0 \cdot 7-2 \cdot 8)\end{array}$ \\
\hline Total & 15 & $8 \cdot 5$ & $1 \cdot 0$ & 29 & $9 \cdot 9$ & $1 \cdot 2(0 \cdot 6-2 \cdot 2)$ & 16 & $10 \cdot 6$ & $1 \cdot 8(0 \cdot 9-3 \cdot 8)$ & 45 & $10 \cdot 2$ & $1 \cdot 5(0 \cdot 6-3 \cdot 7)$ \\
\hline \multicolumn{13}{|c|}{ Degenerative disorders of the myocardium } \\
\hline $\begin{array}{l}\text { Year of first employment: } \\
\quad>1970\end{array}$ & 5 & $4 \cdot 8$ & $1 \cdot 0$ & 4 & $2 \cdot 4$ & $0 \cdot 7(0 \cdot 2-2 \cdot 8)$ & 4 & $4 \cdot 0$ & $1 \cdot 1(0 \cdot 3-4 \cdot 1)$ & 8 & $3 \cdot 0$ & $0.9(0 \cdot 3-2 \cdot 7)$ \\
\hline$\leqslant 1970$ & 2 & $2 \cdot 7$ & $\begin{array}{l}0 \cdot 2(0 \cdot 1-0 \cdot 9) \\
1 \cdot 0\end{array}$ & 9 & $7 \cdot 2$ & $\begin{array}{l}0 \cdot 7(0 \cdot 2-2 \cdot 4) \\
4 \cdot 7(1 \cdot 0-22 \cdot 2)\end{array}$ & 4 & $7 \cdot 8$ & $\begin{array}{l}1 \cdot 2(0 \cdot 3-5 \cdot 1) \\
8 \cdot 3(1 \cdot 5-46 \cdot 4)\end{array}$ & 13 & $7 \cdot 4$ & $\begin{array}{l}0 \cdot 8(0 \cdot 3-2 \cdot 7) \\
5 \cdot 4(1 \cdot 2-24 \cdot 5)\end{array}$ \\
\hline $\begin{array}{l}\text { Duration of employment: } \\
\quad<1 \mathrm{y}\end{array}$ & 2 & $1 \cdot 9$ & $1 \cdot 0$ & 6 & $3 \cdot 5$ & $3 \cdot 2(0 \cdot 6-16 \cdot 6)$ & 6 & $5 \cdot 8$ & $5 \cdot 7(1 \cdot 1-29 \cdot 5)$ & 12 & $4 \cdot 4$ & $4 \cdot 1(0 \cdot 9-19 \cdot 1)$ \\
\hline$\geqslant 1 \mathrm{y}$ & 5 & $7 \cdot 2$ & $\begin{array}{l}2 \cdot 7(0 \cdot 5-13 \cdot 9) \\
1 \cdot 0\end{array}$ & 7 & $5 \cdot 8$ & $\begin{array}{l}3 \cdot 1(0 \cdot 6-15 \cdot 1) \\
1 \cdot 3(0 \cdot 4-4 \cdot 1)\end{array}$ & 2 & $4 \cdot 2$ & $\begin{array}{l}2 \cdot 9(0 \cdot 4-20 \cdot 7) \\
1 \cdot 3(0 \cdot 2-7 \cdot 1)\end{array}$ & 9 & $5 \cdot 3$ & $\begin{array}{l}3 \cdot 0(0 \cdot 6-14 \cdot 3) \\
1 \cdot 3(0 \cdot 4-3 \cdot 9)\end{array}$ \\
\hline $\begin{array}{l}\text { Time since first employment: } \\
<10 \mathrm{y}\end{array}$ & 4 & $3 \cdot 7$ & $1 \cdot 0$ & 2 & $1 \cdot 2$ & $0 \cdot 5(0 \cdot 1-2 \cdot 5)$ & 3 & $3 \cdot 0$ & $1 \cdot 3(0 \cdot 3-5 \cdot 8)$ & 5 & 1.9 & $0 \cdot 7(0 \cdot 2-2 \cdot 8)$ \\
\hline$\geqslant 10 y$ & 3 & $4 \cdot 4$ & $\begin{array}{l}0 \cdot 8(0 \cdot 2-4 \cdot 0) \\
1 \cdot 0\end{array}$ & 11 & 8.9 & $\begin{array}{l}2.6(0.7-9.9) \\
3.5(0.9-13.0)\end{array}$ & 5 & $9 \cdot 8$ & $\begin{array}{l}3 \cdot 1(0 \cdot 7-13 \cdot 8) \\
4 \cdot 7(1 \cdot 0-20 \cdot 4)\end{array}$ & 16 & $9 \cdot 1$ & $\begin{array}{l}2 \cdot 7(0 \cdot 7-10 \cdot 3) \\
3 \cdot 8(1 \cdot 1-13 \cdot 5)\end{array}$ \\
\hline Total & 7 & $4 \cdot 0$ & $1 \cdot 0$ & 13 & $4 \cdot 5$ & $1.7(0.7-4.5)$ & 8 & $5 \cdot 3$ & $2 \cdot 5(0 \cdot 7-5 \cdot 7)$ & 21 & $4 \cdot 7$ & $1.9(0 \cdot 8-4 \cdot 7)$ \\
\hline \multicolumn{13}{|c|}{ Pancreatitis } \\
\hline $\begin{array}{l}\text { Year of first employment: } \\
\quad>1970\end{array}$ & 4 & 3.9 & $1 \cdot 0$ & 7 & $4 \cdot 4$ & $1.0(0.3-3.5)$ & 4 & $3 \cdot 6$ & $0.8(0.2-3 \cdot 4)$ & 11 & $4 \cdot 0$ & $0.9(0 \cdot 3-3 \cdot 0)$ \\
\hline$\leqslant 1970$ & 1 & $1 \cdot 4$ & $\begin{array}{l}0 \cdot 2(0 \cdot 1-1 \cdot 7) \\
1 \cdot 0\end{array}$ & 19 & $14 \cdot 5$ & $\begin{array}{r}2 \cdot 1(0 \cdot 6-6 \cdot 8) \\
11 \cdot 1(1 \cdot 5-8 \cdot 2)\end{array}$ & 2 & $5 \cdot 2$ & $\begin{array}{l}0 \cdot 8(0 \cdot 1-4 \cdot 6) \\
4 \cdot 2(0 \cdot 4-46 \cdot 0)\end{array}$ & 21 & $12 \cdot 4$ & $\begin{array}{l}1 \cdot 8(0 \cdot 5-5 \cdot 7) \\
9 \cdot 6(1 \cdot 3-71 \cdot 0)\end{array}$ \\
\hline $\begin{array}{l}\text { Duration of employment: } \\
\quad<1 \text { y }\end{array}$ & 5 & $4 \cdot 7$ & $1 \cdot 0$ & 18 & $10 \cdot 5$ & $2.4(0.9-6.4)$ & 6 & $5 \cdot 8$ & $1.5(0.4-4.9)$ & 24 & $8 \cdot 7$ & $2 \cdot 1(0 \cdot 8-5 \cdot 5)$ \\
\hline$\geqslant 1 \mathrm{y}$ & 0 & - & - & 8 & $6 \cdot 6$ & $0.9(0.3-2.9)$ & 0 & - & - & 8 & $4 \cdot 8$ & $0 \cdot 7(0 \cdot 2-2 \cdot 2)$ \\
\hline $\begin{array}{l}\text { Time since first employment: } \\
<10 \mathrm{y}\end{array}$ & 1 & $0 \cdot 9$ & $1 \cdot 0$ & 4 & $2 \cdot 4$ & $2 \cdot 7(0 \cdot 3-24 \cdot 0)$ & 3 & $3 \cdot 0$ & $3.4(0.4-32.7)$ & 7 & $2 \cdot 6$ & $2.9(0.4-24 \cdot 0)$ \\
\hline$\geqslant 10 y$ & 4 & $5 \cdot 8$ & $\begin{array}{l}3 \cdot 2(0 \cdot 3-30 \cdot 4) \\
1 \cdot 0\end{array}$ & 22 & $17 \cdot 7$ & $\begin{array}{c}10 \cdot 5(1 \cdot 3-82 \cdot 8) \\
3 \cdot 1(1 \cdot 1-9 \cdot 0)\end{array}$ & 3 & $5 \cdot 9$ & $\begin{array}{l}3 \cdot 7(0 \cdot 4-36 \cdot 8) \\
1 \cdot 1(0 \cdot 2-4 \cdot 9)\end{array}$ & 25 & $14 \cdot 3$ & $\begin{array}{l}8 \cdot 2(1 \cdot 0-63 \cdot 9) \\
2 \cdot 6(0 \cdot 9-7 \cdot 4)\end{array}$ \\
\hline Total & 5 & $2 \cdot 8$ & $1 \cdot 0$ & 26 & $8 \cdot 9$ & $3 \cdot 1(1 \cdot 2-8 \cdot 0)$ & 6 & $4 \cdot 0$ & $1 \cdot 7(0 \cdot 5-5 \cdot 4)$ & 32 & $7 \cdot 2$ & $2 \cdot 6(1 \cdot 0-6 \cdot 8)$ \\
\hline \multicolumn{13}{|c|}{ Glomerulonephritis } \\
\hline $\begin{array}{l}\text { Year of first employment: } \\
>1970\end{array}$ & 0 & - & - & 2 & $1 \cdot 2$ & - & 1 & $0 \cdot 9$ & - & 3 & $1 \cdot 1$ & -- \\
\hline$\leqslant 1970$ & 3 & $4 \cdot 1$ & $1 \cdot 0$ & 3 & $2 \cdot 3$ & $0.6(0 \cdot 1-3 \cdot 2)$ & 2 & $5 \cdot 2$ & $1 \cdot 7(0 \cdot 3-10 \cdot 3)$ & 5 & $2 \cdot 9$ & $0.8(0 \cdot 2-3 \cdot 6)$ \\
\hline $\begin{array}{l}\text { Duration of employment: } \\
<1 \mathrm{y}\end{array}$ & 1 & 0.9 & $1 \cdot 0$ & 2 & $1 \cdot 2$ & $1 \cdot 4(0 \cdot 1-15 \cdot 3)$ & 3 & $2 \cdot 9$ & $4 \cdot 3(0 \cdot 4-42 \cdot 4)$ & 5 & $1 \cdot 8$ & $2 \cdot 3(0 \cdot 3-20 \cdot 1)$ \\
\hline$\geqslant 1 \mathrm{y}$ & 2 & $2 \cdot 9$ & $\begin{array}{l}1 \cdot 9(0 \cdot 2-21 \cdot 2) \\
1 \cdot 0\end{array}$ & 3 & $2 \cdot 5$ & $\begin{array}{l}1.6(0 \cdot 2-15 \cdot 1) \\
0.9(0 \cdot 1-5 \cdot 2)\end{array}$ & $\begin{array}{l}0 \\
0\end{array}$ & -- & - & 3 & $1 \cdot 8$ & $\begin{array}{l}1 \cdot 2(0 \cdot 1-12 \cdot 1) \\
0 \cdot 7(0 \cdot 1-4 \cdot 1)\end{array}$ \\
\hline $\begin{array}{l}\text { Time since first employment: } \\
<10 \mathrm{y}\end{array}$ & 0 & - & - & 2 & $1 \cdot 2$ & - & 1 & $1 \cdot 0$ & - & 3 & $1 \cdot 1$ & - \\
\hline$\underset{\text { Total }}{\geqslant 10 y}$ & $\begin{array}{l}3 \\
3\end{array}$ & $\begin{array}{l}4 \cdot 4 \\
1 \cdot 7\end{array}$ & $\begin{array}{l}1 \cdot 0 \\
1 \cdot 0\end{array}$ & $\begin{array}{l}3 \\
5\end{array}$ & $\begin{array}{l}2 \cdot 4 \\
1 \cdot 7\end{array}$ & $\begin{array}{l}0 \cdot 6(0 \cdot 1-3 \cdot 1) \\
1 \cdot 0(0 \cdot 2-4 \cdot 3)\end{array}$ & $\begin{array}{l}2 \\
3\end{array}$ & $\begin{array}{l}3 \cdot 9 \\
2 \cdot 0\end{array}$ & $\begin{array}{l}1.4(0 \cdot 2-8 \cdot 6) \\
1.7(0 \cdot 3-8 \cdot 8)\end{array}$ & $\begin{array}{l}5 \\
8\end{array}$ & $\begin{array}{l}2 \cdot 9 \\
1 \cdot 8\end{array}$ & $\begin{array}{l}0 \cdot 8(0 \cdot 2-3 \cdot 3) \\
1 \cdot 2(0 \cdot 3-4 \cdot 4)\end{array}$ \\
\hline
\end{tabular}

^Crude mortalities $\left(\times 10^{-5}\right)$.

plastics industry have been thoroughly monitored by the Work Inspection Service since the early 1960 s and have shown a significant decline by calendar year with mean concentrations of 180 parts per million ( $\mathrm{ppm}$ ) during the $1960 \mathrm{~s}$ and $54 \mathrm{ppm}$ during the subsequent decades.

Years at risk were counted from the start of employment (or from the first year the company produced reinforced plastics if later than the start of employment), or 1 January 1970 if employment was before 1970, and continued until death, disappearance, emigration, or 31 December 1990 whichever came first. Follow up for cancer ended on 31 December 1989 as complete cancer registration was not available for later years. Fifty four workers were lost to follow up (0.1\%) and 1072 emigrated during the study period (2\%). A total of 618900 person-years were recorded. According to the national population registry 4484 of the employees died during the follow up. From the national cause of death register the underlying cause and up to two contributory causes of death coded according to the 8th revision of the International Classification of Diseases (ICD-8) were available for all but 94 workers ( 82 of these died abroad). External comparisons were based on the underlying cause, whereas the underlying and the contributory causes were used for internal comparisons (table 1). Multiple sclerosis, parkinsonism, and motor neurone diseases were grouped together with other diseases of the brain under 
Table 4 Solid cancers among men employed in the Danish reinforced plastics industry and similar industries 1970 to 1989 (expected numbers are based on national rates)

\begin{tabular}{|c|c|c|c|c|c|c|}
\hline \multirow[b]{2}{*}{ Solid cancer (ICD-7 code) } & \multicolumn{3}{|c|}{ Reinforced plastic industry } & \multicolumn{3}{|c|}{ Industries with no exposure to styrene } \\
\hline & Obs & $\operatorname{Exp}$ & $\operatorname{SIR}(95 \% C I)$ & $\overline{O b s}$ & $\operatorname{Exp}$ & $\operatorname{SIR}(95 \% C I)$ \\
\hline $\begin{array}{l}\text { All solid cancers (140-199) } \\
\text { Lip (140) } \\
\text { Tongue (141) } \\
\text { Salivary gland (142) } \\
\text { Mouth (143-144) } \\
\text { Pharynx }(145-148) \\
\text { Oesophagus (150) } \\
\text { Stomach (151) } \\
\text { Colon }(153) \\
\text { Rectum }(154) \\
\text { Liver }(155 \cdot 0,156) \\
\text { Gall bladder }(155 \cdot 1) \\
\text { Pancreas }(157) \\
\text { Peritoneum (158, 159) } \\
\text { Nasal cavities (160) } \\
\text { Larynx }(161) \\
\text { Lung }(162 \star, 163) \\
\text { Pleura }(162 \cdot 2) \\
\text { Mediastinum (164) } \\
\text { Prostate (177) } \\
\text { Testis (178) } \\
\text { External male genital organs (179) } \\
\text { Kidney (180) } \\
\text { Urinary bladder (181) } \\
\text { Melanoma of skin (190) } \\
\text { Other skin (191) } \\
\text { Eye (192) } \\
\text { Brain and nervous system (193) } \\
\text { Thyroid (194) } \\
\text { Other endocrinal glands (195) } \\
\text { Bone (196) } \\
\text { Connective tissue (197) } \\
\text { Secondary cancers (198-199) }\end{array}$ & $\begin{array}{r}1134 \\
15 \\
3 \\
5 \\
9 \\
7 \\
13 \\
47 \\
75 \\
47 \\
14 \\
7 \\
41 \\
4 \\
7 \\
25 \\
248 \\
10 \\
3 \\
85 \\
56 \\
7 \\
37 \\
117 \\
26 \\
132 \\
0 \\
46 \\
6 \\
3 \\
3 \\
2 \\
33\end{array}$ & $\begin{array}{r}1147 \cdot 1 \\
14 \cdot 4 \\
4 \cdot 2 \\
2 \cdot 9 \\
8 \cdot 1 \\
10 \cdot 6 \\
14 \cdot 2 \\
47 \cdot 5 \\
76 \cdot 4 \\
59 \cdot 9 \\
19 \cdot 4 \\
7 \cdot 2 \\
34 \cdot 2 \\
3 \cdot 9 \\
3 \cdot 8 \\
22 \cdot 6 \\
222 \cdot 4 \\
5 \cdot 6 \\
1 \cdot 6 \\
86 \cdot 3 \\
55 \cdot 2 \\
4 \cdot 4 \\
39 \cdot 8 \\
101 \cdot 0 \\
36 \cdot 9 \\
163 \cdot 8 \\
3 \cdot 9 \\
47 \cdot 6 \\
4 \cdot 6 \\
1 \cdot 8 \\
3 \cdot 5 \\
5 \cdot 9 \\
26 \cdot 9\end{array}$ & $\begin{array}{l}0.99(0.93-1.05) \\
1.04(0.58-1.71) \\
0.72(0.15-2.09) \\
1.74(0.56-4.05) \\
1.11(0.51-2 \cdot 10) \\
0.66(0.26-1.36) \\
0.92(0.50-1.57) \\
0.99(0.73-1.32) \\
0.98(0.77-1.23) \\
0.78(0.58-1.04) \\
0.72(0.40-1.21) \\
0.97(0.40-1.99) \\
1.20(0.86-1.63) \\
1.03(0.28-2.63) \\
1.84(0.74-3.80) \\
1.10(0.71-1.63) \\
1.12(0.98-1.26) \\
1.78(0.85-3.28) \\
1.84(0.38-5.38) \\
0.98(0.79-1.22) \\
1.02(0.77-1.32) \\
1.60(0.64-3.30) \\
0.93(0.65-1.28) \\
1.16(0.96-1.39) \\
0.70(0.46-1.03) \\
0.81(0.67-0.96) \\
0.00(0.00-0.94) \\
0.97(0.71-1.29) \\
1.31(0.48-2.85) \\
1.66(0.34-4.84) \\
0.87(0.18-2.54) \\
0.34(0.04-1.21) \\
1.22(0.84-1.72)\end{array}$ & $\begin{array}{r}529 \\
9 \\
5 \\
0 \\
1 \\
4 \\
7 \\
33 \\
29 \\
19 \\
5 \\
2 \\
14 \\
1 \\
2 \\
14 \\
123 \\
7 \\
2 \\
45 \\
27 \\
1 \\
19 \\
45 \\
9 \\
70 \\
3 \\
18 \\
0 \\
1 \\
0 \\
2 \\
12\end{array}$ & $\begin{array}{r}507 \cdot 3 \\
6 \cdot 3 \\
1 \cdot 7 \\
1 \cdot 2 \\
3 \cdot 4 \\
4 \cdot 4 \\
6 \cdot 2 \\
21 \cdot 9 \\
34 \cdot 6 \\
27 \cdot 2 \\
8 \cdot 7 \\
3 \cdot 4 \\
15 \cdot 6 \\
1 \cdot 7 \\
1 \cdot 6 \\
9 \cdot 7 \\
99 \cdot 8 \\
2 \cdot 4 \\
0 \cdot 7 \\
42 \cdot 5 \\
22 \cdot 1 \\
1 \cdot 9 \\
17 \cdot 4 \\
45 \cdot 1 \\
14 \cdot 7 \\
70 \cdot 8 \\
1 \cdot 7 \\
19 \cdot 4 \\
1 \cdot 9 \\
0 \cdot 8 \\
1 \cdot 4 \\
2 \cdot 5 \\
11 \cdot 9 \\
\end{array}$ & $\begin{array}{l}1.04(0.96-1.14) \\
1.43(0.65-2.71) \\
2.92(0.95-6.82) \\
0.00(0.00-3.00) \\
0.30(0.01-1.65) \\
0.92(0.25-2.34) \\
1.13(0.45-2.32) \\
1.50(1.03-2.11) \\
0.84(0.56-1.20) \\
0.70(0.42-1.09) \\
0.56(0.19-1.35) \\
0.60(0.07-2 \cdot 16) \\
0.90(0.49-1.51) \\
0.60(0.02-3.34) \\
1.24(0.15-4.49) \\
1.45(0.79-2.43) \\
1.23(1.02-1.47) \\
2.90(1.16-5.96) \\
2.95(0.36-10.62) \\
1.06(0.77-1.42) \\
1.22(0.81-1.78) \\
0.52(0.01-2.90) \\
1.09(0.66-1.70) \\
1.00(0.73-1.33) \\
0.61(0.28-1.16 \\
0.99(0.77-1.25) \\
1.82(0.38-5.31) \\
0.93(0.55-1.47) \\
0.00(0.00-1.92) \\
1.32(0.03-7.33) \\
0.00(0.00-2.60) \\
0.81(0.10-2.93) \\
1.01(0.52-1.77)\end{array}$ \\
\hline
\end{tabular}

*ICD-7 codes $162-162 \cdot 8$, except $162 \cdot 2$.

the heading degenerative disorders of the nervous system, because of the limited knowledge of the possible neurotoxic actions of organic solvents and the few cases within each diagnostic category. For similar reasons, and because of the difficulties in the diagnosis of congestive cardiomyopathy, cardiomyopathy was grouped together with other myocardial insufficiency without additional indications of arteriosclerotic or hypertensive disorder and myocarditis under the heading degenerative disorders of the myocardium.

In the national cancer registry 1663 newly diagnosed cases of solid cancer according to the ICD-7 were identified during the follow up period.

\section{STATISTICAL METHODS}

Standardised mortality ratios (SMRs) and standardised incidence ratios (SIRs) were calculated by comparing the observed number of deaths and newly diagnosed cancers in the cohort with the expected numbers according to age and calendar specific national rates. Poisson regression models were used in internal analyses to estimate mortality rate ratios (MRRs) and incidence rate ratios (IRRs) with the rates for workers unexposed to styrene as the controls. ${ }^{25}$ The Poisson models included the following variables: exposure probability (unexposed, low, high), age (1-49, $50-59,60-69, \geqslant 70$ years), year of first employment $(\leqslant 1970,>1970)$, duration of employment $(<1, \geqslant$ year $)$, and time since first employment $(<10, \geqslant 10$ years). For all analyses $95 \%$ confidence intervals (95\% CIs) are given.

\section{Results}

NON-MALIGNANT DISEASES

The overall mortality for workers in the reinforced plastics industry was increased by $10 \%$ compared with the Danish population at large (table 2). A somewhat larger excess mortality was found in workers of companies with no exposure to styrene, and the workers in the reinforced plastics industry showed a reduced overall mortality when a direct comparison was made (MRR 0.92, 95\% CI 0.87-0.98, data not shown).

The mortality for degenerative disorders of the nervous system defined by the underlying cause of death was close to the national level (table 2). If the case definition instead was based on the underlying and the contributory causes of death and the workers unexposed to styrene were used as controls, an $80 \%$ excess mortality was found in the workers with a high probability of exposure (table 3 ). In this group the mortality was also higher among workers first employed in the 1960s, employed for more than one year, and with a 10 year latency.

If the analysis was restricted to a comparison of workers highly exposed to reinforced plastics with workers not exposed to styrene all workers first employed in the $1960 \mathrm{~s}$, employed for more than one year, or workers with a latent period of 10 years or more after first employment a general pattern of an increased mortality among the workers exposed to reinforced plastics was found.

Degenerative disorders of the myocardium showed an increased SMR in the workers with reinforced plastics industry, whereas a mortality deficit was seen in the workers not exposed 
Table 5 Incidence and incidence rate ratios (IRRs) for cancer of the pancreas, nasal cavities, lung, pleura, external genital organs, and bladder among men employed in the Danish reinforced plastics (RP) industry 1970 to 1989 (results of Poisson regression analysis with workers not exposed to styrene as controls)

\begin{tabular}{|c|c|c|c|c|c|c|c|c|c|c|c|c|}
\hline \multirow{3}{*}{$\begin{array}{l}\text { Cause of death and } \\
\text { employment } \\
\text { data }\end{array}$} & \multicolumn{12}{|c|}{ Exposure probability } \\
\hline & \multicolumn{3}{|c|}{ Unexposed controls } & \multicolumn{3}{|l|}{ Low } & \multicolumn{3}{|l|}{ High } & \multicolumn{3}{|c|}{ All $R P$ workers } \\
\hline & Obs & Rate & $\operatorname{IRR}(95 \% C I)$ & Obs & Rate & $\operatorname{IRR}(95 \% C I)$ & Obs & Rate & $\operatorname{IRR}(95 \% C I)$ & $O b s$ & Rate & $\operatorname{IRR}(95 \% C I)$ \\
\hline \multicolumn{13}{|c|}{ Pancreas } \\
\hline $\begin{array}{l}\text { Year of nirst employment: } \\
>1970\end{array}$ & 4 & $4 \cdot 5$ & $1 \cdot 0$ & 7 & $5 \cdot 1$ & $1 \cdot 0(0 \cdot 3-3 \cdot 3)$ & 7 & $7 \cdot 2$ & $1 \cdot 4(0 \cdot 4-4 \cdot 7)$ & 14 & $6 \cdot 0$ & $1 \cdot 1(0 \cdot 4-3 \cdot 5)$ \\
\hline$\leqslant 1970$ & 10 & $14 \cdot 4$ & $\begin{array}{l}0 \cdot 7(0 \cdot 2-2 \cdot 5) \\
1 \cdot 0\end{array}$ & 17 & $13 \cdot 6$ & $\begin{array}{l}0.9(0 \cdot 3-2 \cdot 8) \\
1 \cdot 1(0 \cdot 5-2 \cdot 5)\end{array}$ & 10 & $27 \cdot 2$ & $\begin{array}{l}2 \cdot 0(0 \cdot 6-6 \cdot 9) \\
2 \cdot 6(1 \cdot 1-6 \cdot 3)\end{array}$ & 27 & $16 \cdot 7$ & $\begin{array}{l}1 \cdot 1(0 \cdot 4-3 \cdot 4) \\
1 \cdot 4(0 \cdot 7-3 \cdot 0)\end{array}$ \\
\hline $\begin{array}{l}\text { Duration of employment: } \\
\quad<1 \mathrm{y}\end{array}$ & 4 & $1 \cdot 1$ & $1 \cdot 0$ & 11 & $7 \cdot 4$ & $2 \cdot 2(0 \cdot 7-6 \cdot 8)$ & 9 & $10 \cdot 1$ & $3 \cdot 1(0 \cdot 9-10 \cdot 3)$ & 20 & $8 \cdot 4$ & $2 \cdot 5(0 \cdot 8-7 \cdot 2)$ \\
\hline$\geqslant 1 \mathrm{y}$ & 10 & $15 \cdot 5$ & $\begin{array}{l}1.9(0 \cdot 6-5 \cdot 9) \\
1.0\end{array}$ & 13 & $11 \cdot 6$ & $\begin{array}{l}1.4(0.5-4 \cdot 4) \\
0.8(0 \cdot 4-1 \cdot 8)\end{array}$ & 8 & $18 \cdot 1$ & $\begin{array}{l}3 \cdot 4(1 \cdot 0-11 \cdot 4) \\
1 \cdot 7(0 \cdot 6-4 \cdot 4)\end{array}$ & 21 & $13 \cdot 4$ & $\begin{array}{l}1 \cdot 8(0 \cdot 6-7 \cdot 4) \\
1 \cdot 0(0 \cdot 5-2 \cdot 1)\end{array}$ \\
\hline $\begin{array}{l}\text { Time since first employment: } \\
<10 \mathrm{y}\end{array}$ & 5 & $5 \cdot 2$ & 1.0 & 7 & $4 \cdot 7$ & $0 \cdot 9(0 \cdot 3-2 \cdot 8)$ & 8 & $9 \cdot 0$ & $2 \cdot 1(0 \cdot 7-6 \cdot 5)$ & 15 & $6 \cdot 3$ & $1 \cdot 3(0 \cdot 5-3 \cdot 5)$ \\
\hline$\geqslant 10 y$ & 9 & $14 \cdot 7$ & $\begin{array}{l}1 \cdot 0(0 \cdot 3-3 \cdot 2) \\
1 \cdot 0\end{array}$ & 17 & $15 \cdot 2$ & $\begin{array}{l}1 \cdot 2(0 \cdot 4-3 \cdot 7) \\
1 \cdot 3(0 \cdot 6-2 \cdot 8)\end{array}$ & 9 & $20 \cdot 3$ & $\begin{array}{l}2 \cdot 1(0 \cdot 7-6 \cdot 9) \\
2 \cdot 0(0 \cdot 8-5 \cdot 2)\end{array}$ & 26 & $16 \cdot 7$ & $\begin{array}{l}1 \cdot 5(0 \cdot 5-4 \cdot 3) \\
1 \cdot 4(0 \cdot 7-3 \cdot 1)\end{array}$ \\
\hline Total & 14 & $8 \cdot 9$ & $1 \cdot 0$ & 24 & $9 \cdot 2$ & $1 \cdot 1(0 \cdot 6-2 \cdot 2)$ & 17 & $12 \cdot 7$ & $2 \cdot 2(1 \cdot 1-4 \cdot 5)$ & 41 & - & $1 \cdot 4(0 \cdot 8-2 \cdot 6)$ \\
\hline \multicolumn{13}{|c|}{ Nasal cavities } \\
\hline$>1970$ & 0 & - & - & 4 & $2 \cdot 9$ & - & 1 & $1 \cdot 0$ & - & 5 & $2 \cdot 1$ & - \\
\hline $\begin{array}{l}\quad \leqslant 1970 \\
\text { Duration of employment: }\end{array}$ & 2 & $2 \cdot 9$ & $1 \cdot 0$ & 1 & $0 \cdot 8$ & $0 \cdot 3(0 \cdot 1-3 \cdot 2)$ & 1 & $2 \cdot 7$ & $1 \cdot 0(0 \cdot 1-11 \cdot 2)$ & 2 & $1 \cdot 2$ & $0 \cdot 4(0 \cdot 1-3 \cdot 2)$ \\
\hline$<1 \mathrm{y}$ & 0 & - & - & 3 & $2 \cdot 0$ & - & 1 & $1 \cdot 1$ & - & 4 & $1 \cdot 7$ & - \\
\hline $\begin{array}{l}\geqslant 1 \mathrm{y} \\
\text { Time since first employment: }\end{array}$ & 2 & $3 \cdot 2$ & $1 \cdot 0$ & 2 & $1 \cdot 8$ & $0 \cdot 5(0 \cdot 1-3 \cdot 7)$ & 1 & $2 \cdot 3$ & $0 \cdot 8(0 \cdot 1-8 \cdot 5)$ & 3 & $2 \cdot 0$ & $0 \cdot 6(0 \cdot 1-3 \cdot 5)$ \\
\hline$<10 y$ & 0 & - & - & 2 & $1 \cdot 3$ & - & 2 & $2 \cdot 2$ & - & 4 & $1 \cdot 7$ & - \\
\hline$\geqslant 10 y$ & 2 & $3 \cdot 3$ & $1 \cdot 0$ & 3 & $2 \cdot 7$ & $0 \cdot 8(0 \cdot 1-5 \cdot 1)$ & 0 & - & - & 3 & 1.9 & $0.6(0 \cdot 1-3 \cdot 5)$ \\
\hline Total & 2 & $1 \cdot 3$ & $1 \cdot 0$ & 5 & 1.9 & $1.5(0.3-8 \cdot 0)$ & 2 & 1.5 & $1 \cdot 3(0 \cdot 2-9 \cdot 3)$ & 7 & $1 \cdot 8$ & $1.5(0 \cdot 3-7 \cdot 1)$ \\
\hline \multicolumn{13}{|c|}{ Lung } \\
\hline$>1970$ & 26 & $29 \cdot 3$ & $1 \cdot 0$ & 46 & $33 \cdot 7$ & $1 \cdot 0(0 \cdot 6-1 \cdot 6)$ & 40 & $41 \cdot 3$ & $1 \cdot 3(0 \cdot 8-2 \cdot 1)$ & 86 & $36 \cdot 9$ & $1 \cdot 1(0 \cdot 7-1 \cdot 7)$ \\
\hline$\leqslant 1970$ & 97 & $139 \cdot 3$ & $\begin{array}{l}1 \cdot 8(1 \cdot 1-3 \cdot 0) \\
1 \cdot 0\end{array}$ & 130 & $104 \cdot 2$ & $\begin{array}{l}1.6(0.9-2 \cdot 5) \\
0.9(0 \cdot 7-1 \cdot 1)\end{array}$ & 32 & $87 \cdot 2$ & $\begin{array}{l}1 \cdot 5(0 \cdot 9-2 \cdot 6) \\
0.8(0 \cdot 5-1 \cdot 2)\end{array}$ & 162 & $100 \cdot 4$ & $\begin{array}{l}1.6(0.9-2 \cdot 5) \\
0.9(0.7-1 \cdot 1)\end{array}$ \\
\hline $\begin{array}{l}\text { Duration of employment: } \\
\quad<1 \mathrm{y}\end{array}$ & 49 & $51 \cdot 3$ & $1 \cdot 0$ & 70 & $46 \cdot 5$ & $1 \cdot 0(0 \cdot 7-1 \cdot 5)$ & 52 & $57 \cdot 4$ & $1 \cdot 4(0 \cdot 9-2 \cdot 1)$ & 122 & $50 \cdot 6$ & $1 \cdot 2(0 \cdot 8-1 \cdot 6)$ \\
\hline$\geqslant 1 \mathrm{y}$ & 74 & $117 \cdot 8$ & $\begin{array}{l}1 \cdot 2(0 \cdot 9-1 \cdot 8) \\
1 \cdot 0\end{array}$ & 106 & $95 \cdot 9$ & $\begin{array}{l}1 \cdot 0(0 \cdot 7-1 \cdot 4) \\
0 \cdot 8(0 \cdot 6-1 \cdot 1)\end{array}$ & 20 & $46 \cdot 5$ & $\begin{array}{l}0.7(0.4-1 \cdot 2) \\
0.6(0.3-0.9)\end{array}$ & 126 & $82 \cdot 1$ & $\begin{array}{l}0.9(0 \cdot 7-1 \cdot 3) \\
0.8(0.6-1 \cdot 0)\end{array}$ \\
\hline $\begin{array}{l}\text { Time since first employment: } \\
<10 \mathrm{y}\end{array}$ & 40 & $41 \cdot 3$ & $1 \cdot 0$ & 52 & $34 \cdot 8$ & $0 \cdot 8(0 \cdot 6-1 \cdot 3)$ & 26 & $29 \cdot 1$ & $0.8(0.5-1.4)$ & 78 & $32 \cdot 7$ & $0 \cdot 8(0 \cdot 6-1 \cdot 2)$ \\
\hline$\geqslant 10 \mathrm{y}$ & 83 & $135 \cdot 4$ & $\begin{array}{l}1 \cdot 0(0 \cdot 6-1 \cdot 6) \\
1 \cdot 0\end{array}$ & 124 & $111 \cdot 1$ & $\begin{array}{l}0.9(0.6-1 \cdot 4) \\
0.9(0.7-1 \cdot 2)\end{array}$ & 46 & 103.9 & $\begin{array}{l}1 \cdot 1(0 \cdot 6-1 \cdot 7) \\
1 \cdot 1(0 \cdot 7-1 \cdot 5)\end{array}$ & 170 & $109 \cdot 0$ & $\begin{array}{l}0.9(0 \cdot 6-1 \cdot 5) \\
0.9(0 \cdot 7-1 \cdot 2)\end{array}$ \\
\hline Total & 123 & $77 \cdot 7$ & $1 \cdot 0$ & 176 & $67 \cdot 4$ & $0.9(0 \cdot 7-1 \cdot 1)$ & 72 & $53 \cdot 9$ & $1 \cdot 0(0 \cdot 7-1 \cdot 3)$ & 248 & $62 \cdot 8$ & $0.9(0 \cdot 7-1 \cdot 1)$ \\
\hline \multicolumn{13}{|c|}{ Pleura } \\
\hline$>1970$ & 0 & - & - & 2 & 1.5 & - & 3 & $3 \cdot 1$ & - & 5 & $2 \cdot 1$ & - \\
\hline $\begin{array}{l}\quad \leqslant 1970 \\
\text { Duration of employment: }\end{array}$ & 7 & $10 \cdot 1$ & $1 \cdot 0$ & 2 & $1 \cdot 6$ & $0 \cdot 2(0 \cdot 1-0 \cdot 8)$ & 3 & $8 \cdot 1$ & $1 \cdot 0(0 \cdot 2-3 \cdot 7)$ & 5 & $3 \cdot 1$ & $0 \cdot 3(0 \cdot 1-1 \cdot 1)$ \\
\hline$<1 \mathrm{y}$ & 0 & - & - & 1 & $0 \cdot 7$ & - & 5 & $5 \cdot 5$ & - & 6 & $2 \cdot 5$ & - \\
\hline $\begin{array}{l}\geqslant 1 \mathrm{y} \\
\text { Time since first employment: }\end{array}$ & 7 & $11 \cdot 1$ & $1 \cdot 0$ & 3 & $2 \cdot 7$ & $0 \cdot 2(0 \cdot 1-0 \cdot 9)$ & 1 & $2 \cdot 3$ & $0 \cdot 2(0 \cdot 1-1 \cdot 9)$ & 4 & $2 \cdot 6$ & $0 \cdot 2(0 \cdot 1-0 \cdot 8)$ \\
\hline$<10 y$ & 0 & - & - & 2 & $1 \cdot 3$ & - & 1 & $1 \cdot 1$ & - & 3 & $1 \cdot 3$ & - \\
\hline$\geqslant 10 \mathrm{y}$ & 7 & $11 \cdot 4$ & $1 \cdot 0$ & 2 & $1 \cdot 8$ & $0 \cdot 2(0 \cdot 1-0 \cdot 8)$ & 5 & $11 \cdot 3$ & $1 \cdot 2(0 \cdot 4-3 \cdot 8)$ & 7 & $4 \cdot 5$ & $0 \cdot 4(0 \cdot 1-1 \cdot 2)$ \\
\hline Total & 7 & $4 \cdot 4$ & $1 \cdot 0$ & 4 & 1.5 & $0 \cdot 4(0 \cdot 1-1 \cdot 3)$ & 6 & $4 \cdot 5$ & $1 \cdot 6(0 \cdot 5-4 \cdot 9)$ & 10 & $2 \cdot 5$ & $0 \cdot 7(0 \cdot 3-1 \cdot 8)$ \\
\hline \multicolumn{13}{|c|}{ External male genital organs } \\
\hline$>1970$ & 0 & - & - & 1 & $1 \cdot 4$ & 1 & 0 & - & - & 1 & $0 \cdot 4$ & - \\
\hline $\begin{array}{l}\quad \leqslant 1970 \\
\text { Duration of employment: }\end{array}$ & 1 & $1 \cdot 4$ & $1 \cdot 0$ & 6 & $4 \cdot 8$ & $3.5(0.4-29 \cdot 0)$ & 0 & - & - & 6 & $3 \cdot 7$ & $2 \cdot 7(0 \cdot 3-22 \cdot 7)$ \\
\hline$<1 \mathrm{y}$ & 0 & - & $-(-)$ & 1 & $0 \cdot 7$ & $-(-)$ & 0 & - & - & 1 & $1 \cdot 0$ & - \\
\hline $\begin{array}{l}\geqslant 1 \mathrm{y} \\
\text { Time since first employment: }\end{array}$ & 1 & $1 \cdot 6$ & $1 \cdot 0$ & 6 & $5 \cdot 4$ & $3.5(0 \cdot 4-29 \cdot 4)$ & 0 & - & - & 6 & $3 \cdot 9$ & $2 \cdot 5(0 \cdot 3-20 \cdot 8)$ \\
\hline$<10 y$ & 0 & - & $-(-)$ & 3 & $2 \cdot 0$ & $-(-)$ & 0 & - & - & 3 & $1 \cdot 3$ & - \\
\hline$\geqslant 10 \mathrm{y}$ & 1 & $1 \cdot 6$ & $1 \cdot 0$ & 4 & $3 \cdot 6$ & $2 \cdot 2(0 \cdot 2-19 \cdot 8)$ & 0 & - & - & 4 & 2.5 & $1 \cdot 6(0 \cdot 2-14 \cdot 2)$ \\
\hline Total & 1 & $0 \cdot 6$ & $1 \cdot 0$ & 7 & $2 \cdot 7$ & $4 \cdot 3(0 \cdot 5-35 \cdot 0)$ & 0 & - & - & 7 & 1.8 & $3 \cdot 0(0 \cdot 4-24 \cdot 2)$ \\
\hline \multicolumn{13}{|c|}{ Urinary bladder } \\
\hline $\begin{array}{l}\text { Year of tirst employment: } \\
\quad>1970\end{array}$ & 10 & $11 \cdot 3$ & $1 \cdot 0$ & 25 & $18 \cdot 3$ & $1 \cdot 4(0 \cdot 7-2 \cdot 9)$ & 18 & $18 \cdot 6$ & $1 \cdot 4(0 \cdot 7-3 \cdot 1)$ & 43 & $18 \cdot 4$ & $1.4(0 \cdot 7-2 \cdot 8)$ \\
\hline$\leqslant 1970$ & 35 & $50 \cdot 3$ & $\begin{array}{l}1 \cdot 3(0 \cdot 6-2 \cdot 8) \\
1 \cdot 0\end{array}$ & 58 & $46 \cdot 5$ & $\begin{array}{l}1.4(0 \cdot 7-2 \cdot 9) \\
1 \cdot 1(0 \cdot 7-1 \cdot 7)\end{array}$ & 16 & $43 \cdot 6$ & $\begin{array}{l}1 \cdot 6(0 \cdot 7-3 \cdot 6) \\
1 \cdot 2(0 \cdot 7-2 \cdot 1)\end{array}$ & 74 & $45 \cdot 8$ & $\begin{array}{l}1.5(0.7-2 \cdot 9) \\
1 \cdot 1(0 \cdot 7-1.7)\end{array}$ \\
\hline $\begin{array}{l}\text { Duration of employment: } \\
\quad<1 \mathrm{y}\end{array}$ & 15 & $15 \cdot 7$ & $1 \cdot 0$ & 30 & $19 \cdot 9$ & $1 \cdot 4(0 \cdot 8-2 \cdot 6)$ & 15 & $16 \cdot 5$ & $1 \cdot 3(0 \cdot 6-2 \cdot 7)$ & 45 & $18 \cdot 7$ & $1 \cdot 4(0 \cdot 8-25)$ \\
\hline$\geqslant 1 \mathrm{y}$ & 30 & $47 \cdot 8$ & $\begin{array}{l}1 \cdot 6(0 \cdot 9-3 \cdot 0) \\
1 \cdot 0\end{array}$ & 53 & $47 \cdot 9$ & $\begin{array}{l}1 \cdot 6(0 \cdot 9-2 \cdot 8) \\
1 \cdot 1(0 \cdot 6-1 \cdot 6)\end{array}$ & 19 & $44 \cdot 2$ & $\begin{array}{l}2 \cdot 1(1 \cdot 1-4 \cdot 1) \\
1 \cdot 3(0 \cdot 8-2 \cdot 4)\end{array}$ & 72 & $46 \cdot 9$ & $\begin{array}{l}1 \cdot 7(0 \cdot 9-3 \cdot 0) \\
1 \cdot 1(0 \cdot 7-1 \cdot 6)\end{array}$ \\
\hline $\begin{array}{l}\text { Time since first employment: } \\
<10 \mathrm{y}\end{array}$ & 11 & $11 \cdot 3$ & $1 \cdot 0$ & 25 & $16 \cdot 7$ & $1 \cdot 4(0 \cdot 7-2 \cdot 8)$ & 13 & $14 \cdot 5$ & $1.5(0 \cdot 7-3 \cdot 3)$ & 38 & $15 \cdot 9$ & $1 \cdot 4(0 \cdot 7-2 \cdot 8)$ \\
\hline$\geqslant 10 \mathrm{y}$ & 34 & $55 \cdot 5$ & $\begin{array}{l}1 \cdot 5(0 \cdot 7-3 \cdot 1) \\
1 \cdot 0\end{array}$ & 58 & $51 \cdot 9$ & $\begin{array}{l}1 \cdot 5(0 \cdot 8-3 \cdot 1) \\
1 \cdot 1(0 \cdot 7-1 \cdot 6)\end{array}$ & 21 & $47 \cdot 4$ & $\begin{array}{l}1 \cdot 7(0 \cdot 8-3 \cdot 7) \\
1 \cdot 7(0 \cdot 7-2 \cdot 0)\end{array}$ & 79 & $50 \cdot 7$ & $\begin{array}{l}1 \cdot 6(0 \cdot 8-3 \cdot 2) \\
1 \cdot 1(0 \cdot 7-1 \cdot 6)\end{array}$ \\
\hline Total & 45 & $28 \cdot 4$ & $1 \cdot 0$ & 83 & $31 \cdot 8$ & $1 \cdot 1(0 \cdot 8-1 \cdot 6)$ & 34 & $25 \cdot 4$ & $1 \cdot 2(0.8-2 \cdot 0)$ & 117 & $29 \cdot 6$ & $1.2(0.8-1.6)$ \\
\hline
\end{tabular}

${ }^{\star}$ Crude incidence $\left(\times 10^{-5}\right)$. 
(table 2). In the internal analysis, the mortality increased with probability of exposure and a higher mortality was indicated more than 10 years after the start of employment in the reinforced plastics industry (table 3). Among the workers in the reinforced plastics industry, the short term workers showed the highest mortality and the increased mortality for long term workers vanished when long term workers not exposed to styrene were selected as controls. Cardiomyopathy showed an MRR of $2 \cdot 2$ (five observed, $95 \%$ CI $0 \cdot 5-9 \cdot 3$, data not shown) among workers with a high probability of exposure, and among these workers the MRR was $5 \cdot 2$ (four observed, 95\% CI $0 \cdot 8-35 \cdot 6$ ) more than 10 years after start of employment. But the increased mortality found was entirely confined to short term workers.

The mortality for pancreatitis showed a slightly increased SMR (table 2), which corresponded to a doubled MRR in the internal analysis (table 3 ). But the mortality declined with probability of exposure to styrene and the excess mortality was confined to the short term workers. Among workers with low probability of exposure much higher mortality was found among workers of the 1960s and more than 10 years after the start of employment. This pattern was, on the other hand, not apparent in the employees with high probability of exposure. Twenty of the exposed cases had a diagnosis of chronic pancreatitis opposed to only one case among the nonexposed workers.

The SMR for glomerulonephritis among workers in the reinforced plastics industry did not deviate from one (table 2). Only three cases occurred among workers in the reinforced plastics industry with high probability of exposure (table 3 ). This number corresponds to a small increase in mortality, but all three workers had been employed for less than one year in the industry. The SMRs for symptoms, ill defined conditions, accidents, poisonings, and violence (including suicide) were moderately increased for the workers in the reinforced plastics industry. Comparable SMRs were found for the workers not exposed to styrene, and for this reason and because these outcomes were not expected to be associated with exposure to styrene no further evaluation by internal analysis was made.

\section{MALIGNANT DISEASES}

The total number of solid cancers found among the workers in the reinforced plastics industry and the employees of companies with no exposure to styrene were identical to the expected values calculated from national rates (table 4). Cancer of the salivary glands, the pancreas, the nasal cavities, the lungs, the pleura (mesothelioma), the mediastinum, the external male genital organs, and the bladder all showed increased SIRs.

Five cases of cancer of the salivary glands were found and all started employment in the reinforced plastics industry in the 1960 s, which corresponded to an SIR of 3.16 for this subgroup of workers $(95 \%$ CI $1 \cdot 03-7 \cdot 39$, data not shown). The increased risk was, however, confined to short term workers. No case occurred among the workers not exposed to styrene. The other cancers with increased incidence in the external analysis were further studied by Poisson modelling with the unexposed workers as controls (except for cancer of the mediastinum because of the few cases).

In the internal analysis pancreatic cancer showed the highest risk among workers with the highest probability of exposure and among these workers the risk was highest among long term workers and workers who started employment in a reinforced plastics company during the 1960s (table 5). If the comparison was restricted to workers who started employment during the 1960 s, or to long term workers, or to the person years accumulated 10 years or more after the start of employment, the general picture of an increased occurrence among the workers in the reinforced plastics industry remained.

Cancer of the nasal cavities showed a slightly increased risk in the internal analysis for workers in the reinforced plastics industry (table 5). The analysis was limited by the small number of cases, and there was no indication that risk increased with increasing probability of exposure to styrene, first year of employment, or duration of employment.

The IRR for cancer of the external male genital organs was three times higher among workers in the reinforced plastics industry than the workers not exposed to styrene, but all the seven exposed cases occurred among workers with a low probability of exposure (table 5). Of these seven cases, six were cancer of the penis and one was a scrotal cancer. The single unexposed case was also a scrotal cancer.

Lung cancer, cancer of the pleura, and cancer of the bladder showed no increased risk among the workers of the reinforced plastics industry compared with the workers not exposed to styrene (table 5). Taking the year of first employment, duration of employment, or time since first employment into consideration did not change these patterns.

\section{Discussion}

Neurological impairment ranging from mild temporary symptoms to severe chronic disease has been associated with occupational exposure to organic solvents. Styrene has been linked to several of these adverse health effects. The neurotoxic mechanisms of organic solvents are, however, still unknown and for this reason cases of parkinsonism, multiple sclerosis, unspecified degenerative disorders of the brain, and motor neurone disease were aggregated in spite of the fact that the pathologies of the diseases are obviously different. This combined category of diseases occurred more often among the workers in the reinforced plastics industry in the internal analysis. No excess mortality compared with the Danish population was seen, but the comparisons were based on small numbers.

Degenerative disorders of the myocardium 
had an increased SMR. This result occurred in the internal analysis mainly because of the short term workers. Congestive cardiomyopathy, the main disease we were originally interested in, could not be separated from other categories of cardiomyopathy. The mortality from ischaemic heart disease was not increased among the workers in the reinforced plastics industry (MRR 0.90, 932 cases, $95 \%$ CI $0.81-1.01$, data not shown), which is in line with most earlier studies of workers exposed to organic solvents other than carbon disulphide. ${ }^{26}$

The workers in the reinforced plastics industry had 10 deaths due to pancreatitis compared with 7.9 expected. Thirty two workers in the reinforced plastics industry had a diagnosis of pancreatitis when both the underlying and the contributing causes of death were considered (SMR 1.52, 95\% CI $1 \cdot 07-2 \cdot 15$, data not shown). The internal analyses did not show any clear associations with the variables that indicated increasing exposure to styrene. Of special importance is the fact that the increased mortality was confined to short term workers.

The slightly increased risk of pancreatic cancer found is in line with the results of two current mortality studies of workers in the reinforced plastics industry in Europe and the United States ${ }^{2022}$ (part of the Danish study population was included in the European multicentre study, but the increased mortality for pancreatic cancer was also found in other subcohorts). Methylene chloride has been linked with pancreatic cancer, ${ }^{27}$ but has been used in only a small proportion of the reinforced plastics companies, ${ }^{3}$ and can hardly explain the findings.

An increased incidence of cancer of the salivary glands was found based on only five cases. This disease has been associated with radiation, but the causes of most cases are unknown. ${ }^{28}$ Nasal cancers are strongly related to exposure to hard wood dust and this is the most likely explanation for the increased risk found in both the reinforced plastics industry and the control industries because of the high proportion of boat yards in both categories. The SIR analysis showed increased risks for cancer of the lung and pleura, which was not found in the internal comparisons. It may indicate similar frequencies of exposure to asbestos and smoking in these industries. The risk pattern of the external male genital organs did not give any strong support for an association with employment in the reinforced plastics industry.

An important limitation is the lack of information on individual exposure. We think that this problem is not serious in our study of the reinforced plastics industry, because the number of exposures are limited and dominated by styrene, which is highly volatile. The industry, especially the companies classified with a high probability of exposure, has also been characterised by rather small companies with few employees. This leaves few opportunities for the employees to avoid styrene expo- sure completely, even when not directly engaged in its production. It is well documented that employment in the reinforced plastics industry during the 1960 s implied high exposure to styrene, so calendar year may be the most valid indicator of level of exposure. Duration of employment together with probability of exposure and year of first employment in the reinforced plastics industry probably gives a valid indication of exposure to styrene.

Confounding by lifestyle factors and especially occupational exposures in other work tasks is a limitation, as no information on such factors was available. The increased overall mortality found for employees of the reinforced plastics industry compared with the Danish population at large is likely to reflect confounding by social class as a high proportion of the study population were unskilled workers known to experience an excess mortality. ${ }^{29}$ For this reason internal comparisons were made with a population similar to the workers in the reinforced plastics industry but without exposure to styrene and these analyses are expected to be the most valid.

The risk of pancreatic cancer is associated with smoking ${ }^{30}$ and confounding by smoking could explain the increased occurrence of pancreatic cancer found among the workers in the reinforced plastics industry. Should that be the case one would expect an increased risk of lung cancer and this was not found.

Pancreatitis and myocardial insufficiency are known to be linked to excess alcohol intake. $^{31} 32$ Mortality from alcoholism and cirrhosis of the liver showed a significantly reduced mortality in long term workers compared with short term workers (MRR, alcoholism 0.3, 267 observed, 95\% CI $0 \cdot 2-0 \cdot 4 ;$ MRR, cirrhosis of the liver 0.3, 38 observed, $95 \%$ CI $0 \cdot 2-0 \cdot 4$, data not shown). These findings thus suggest a higher alcohol intake among the short term workers and is in line with former reports suggesting that short term workers have less favourable life style factors than long term workers. ${ }^{33}$ Even without individual alcohol data it seems reasonable to conclude that confounding by alcohol may explain the increased mortality found for degenerative disorders of the myocardium and pancreatitis in the short term workers in the reinforced plastics industry.

The modification of the internal risk estimates observed in tables 3 and 5 when comparisons were made between exposed and non-exposed workers within similar categories of duration of employment, time since first employment, and year of first employment may be due to secular changes in potential confounders.

In summary these patterns of mortality for degenerative disorders of the nervous system and the incidence of pancreatic cancer give some support to the hypothesis that their occurrence is increased among the workers in the reinforced plastics industry. These findings are in line with previous reports of workers exposed to styrene or other hydrocarbons. All 
the conclusions of this study are of low precision and our findings could easily be explained by chance. No consistent indications of increased occurrence of other nonmalignant diseases or solid cancers were found. Because only company information on exposure was available and because of the lack of individual information on possible confounding factors one has to be careful in interpreting the results. The suggestion of an increase in degenerative disorders of the nervous system and pancreatic cancer deserves in depth studies. This study was supported by the Health Fund, Aarhus University Research Foundation, the Danish Research Academy, the Danish

1 Pfäffli P, Säämänen A. The occupational scene of styrene. In: Sorsa M, Peltonen K, Vainio H, Hemminki K, eds. In: Sorsa $M$, Peltonen $K$, Vainio $\mathrm{H}$, Hemmink $\mathrm{K}$, eds. Butadiene and styrene: assessment of health hazards. Lyon: International Agency for Research on
1993:15-26. (IARC Sci Publ No 127.)

2 Newhook R, Caldwell I. Exposure to styrene in the general Canadian population. In: Sorsa M, Peltonen K, Vainio $\mathrm{H}$, Hemminki $\mathrm{K}$, eds. Butadiene and styrene: assessment of health hazards. Lyon: International Agency for Research on Cancer (IARC), 1993:27-33. (IARC Sci Publ No 127.)

3 Jensen AA, Breum NO, Bacher J, Lynge E. Occupational exposures to styrene in Denmark 1955-88. Am $\mathcal{F}$ Ind Med 1990;17:593-606.

4 Edling C, Anundi H, Johanson G, Nilsson K. Increase in neuropsychiatric symptoms after occupational exposure to low levels of styrene. Br F Ind Med 1993;50:843-50.

5 Matikainen E, Forsman-Grönholm L, Pfäffli P, Juntunen J. Neurotoxicity in workers exposed to styrene. In: Sorsa $\mathrm{M}$, Peltonen $\mathrm{K}$, Vainio $\mathrm{H}$, Hemminki $\mathrm{K}$, eds. Butadiene and styrene: assessment of health hazards. Lyon: International Agency for Research on Cancer (IARC), 1993:153-61. (IARC Sci Publ No 127.)

6 Cherry N, Rodgers B, Venables H, Waldron HA, Wells GG. Acute behavioural effects of styrene exposure: a further analysis. Br f Ind Med 1981;38:346-50.

7 Gobba F, Galassi C, Imbriani M, Ghittori S, Candela S, Cavalleri A. Acquired dyschromatopsia among styreneexposed workers. $₹$ Occup Med 1991;33:761-5.

8 Triebig G, Lehrl S, Weltle D, Schaller KH, Valentin H. Clinical and neurobehavioural study of the acute and chronic neurotoxicity of styrene. Br $\mathcal{F}$ Ind Med 1989; 46:799-804.

9 Landtblom A-M, Flodin U, Karlsson M, Pålhagen S, Axelson $\mathrm{O}$, Söderfeldt B. Multiple sclerosis and expo-
sure to solvents, ionizing radiation and animals. Scand $\mathcal{F}$ sure to solvents, ionizing radiation and

10 Tanner CM. Occupational and environmental causes of parkinsonism. Occup Med 1992;7:503-13.

11 Hawkes CH, Cavanagh JB, Fox AJ. Motorneuron disease: a disorder secondary to solvent exposure? Lancet 1989; i:73-6.

12 Olsen J, Sabroe S. A case-referent study of neuropsychiatric disorders among workers exposed to solvents in the
Danish wood and furniture industry. Scand $\mathcal{f}$ Soc Med 1980;(suppl 16):44-9.

13 Wiseman MN, Banim S. "Glue sniffer's" heart? $B M \mathcal{F}$ 1987;294:739.

14 McNamee $R$ Braganza JM, Hogg J, Leck I, Rose $P$, Cherry NM. Occupational exposure to hydrocarbons and chronic pancreatitis: a case-referent study. Occup and chronic pancreatitis: a

15 Ravnskov U, Lundström S, Nordén A. Hydrocarbon exposure and glomerulonephritis: evidence from patients' occupations. Lancet 1983;ii:1214-6.

16 Porro A, Lomonte C, Coratelli P, Passavanti G, Ferri GM, Assennato $\mathrm{G}$. Chronic glomerulonephritis and exposure to solvents: a case-referent study. Br $\mathcal{F}$ Ind Med 1992; 49:738-42.

17 Harrington JM, Whitby H, Gray CN, Reid FJ, Aw TC, Waterhouse JA. Renal disease and occupational exposure to organic solvents: a case referent approach. Br ₹ Ind Med 1989;46:643-50.

18 Barale $R$. The genetic toxicology of styrene and styrene oxide. Mutat Res 1991;257:107-26.

19 Bond GG, Bodner KM, Olsen GW, Cook RR. Mortality among workers engaged in the development or manufacamong workers engaged in the development or manufacture of styrene-based products-an
Work Environ Health 1992;18:145-54

20 Kogevinas M, Ferro G, Andersen A, Bellander T, Biocca $\mathrm{M}$, Coggon $\mathrm{D}$, et al. Cancer mortality in a historical cohort study of workers exposed to styrene. Scand $\mathcal{F}$ Work Environ Health 1994;20:251-61.

21 Kolstad HA, Lynge E, Olsen J, Breum N. Incidence of lymphohematopoietic malignancies among styreneexposed workers of the reinforced plastics industry. Scand 7 Work Environ Health 1994;20:272-8.

22 Wong $O$, Trent $L S$, Whorton MD. An updated cohort mortality study of workers exposed to styrene in the remortality study of workers exposed to styrene in the reMed 1994;51:386-96.

23 International Agency for Research on Cancer. Some industrial chemicals. LARC Monogr Eval Carcinog Risk Hum 1994;60:233-320

24 Kolstad HA, Lynge E, Olsen J. Cancer incidence in the Danish reinforced plastics industry. In: Sorsa $\mathbf{M}$, Peltonen K, Vainio H, Hemminki K, eds. Butadiene and styrene: assessment of health hazards. Lyon: International Agency for Research on Cancer (IARC), 1993:301-8. (IARC Sci Publ No 127.)

25 Breslow NE, Day NE. Statistical methods in cancer research; vol II. The design and analysis of cohort studies. Lyon: International Agency for Research on Cancer (IARC), 1987. (IARC Sci Publ No 82.)

26 Kristensen TS. Cardiovascular diseases and the work environment. A critical review of the epidemiologic literature
on chemical factors. Scand $\mathcal{F}$ Work Environ Health 1989; on chemical

27 Hearne FT, Pifer JW, Grose F. Absence of adverse mortality effects in workers exposed to methylene chloride: an update. $₹$ Occup Med 1990;32:234-40.

28 Kolstad HA, Lynge E, Olsen J, Sabroe S. Occupational causes of some rare cancers. A literature review. Scand $\mathcal{F}$ Social Med 1992;(suppl 48):1-148.

29 Lynge E. Occupational mortality. Copenhagen: Danmarks Statistik, 1979 (in Danish).

30 International Agency for Research on Cancer. Tobacco smoking. LARC Monogr Eval Carcinog Risk Hum 1986; 38:279-82.

31 Yen S, Chung-Cheng H, MacMahon B. Consumption of alcohol and tobacco and other risk factors for pancreatitis. alcohol and tobacco and other risk

32 Urbano-Marquez A, Estruch R, Navarro-Lopez F, Grau JM, Mont L, Rubin E. The effects of alcoholism on skeletal and cardial muscle. $N$ Engl $f$ Med 1989;320: 409-15.

33 Gilbert ES. Some confounding factors in the study of mortality and occupational exposures. Am f Epidemiol 1982; 116: 177-88. 